\title{
Interview with Dr Bowsher
}
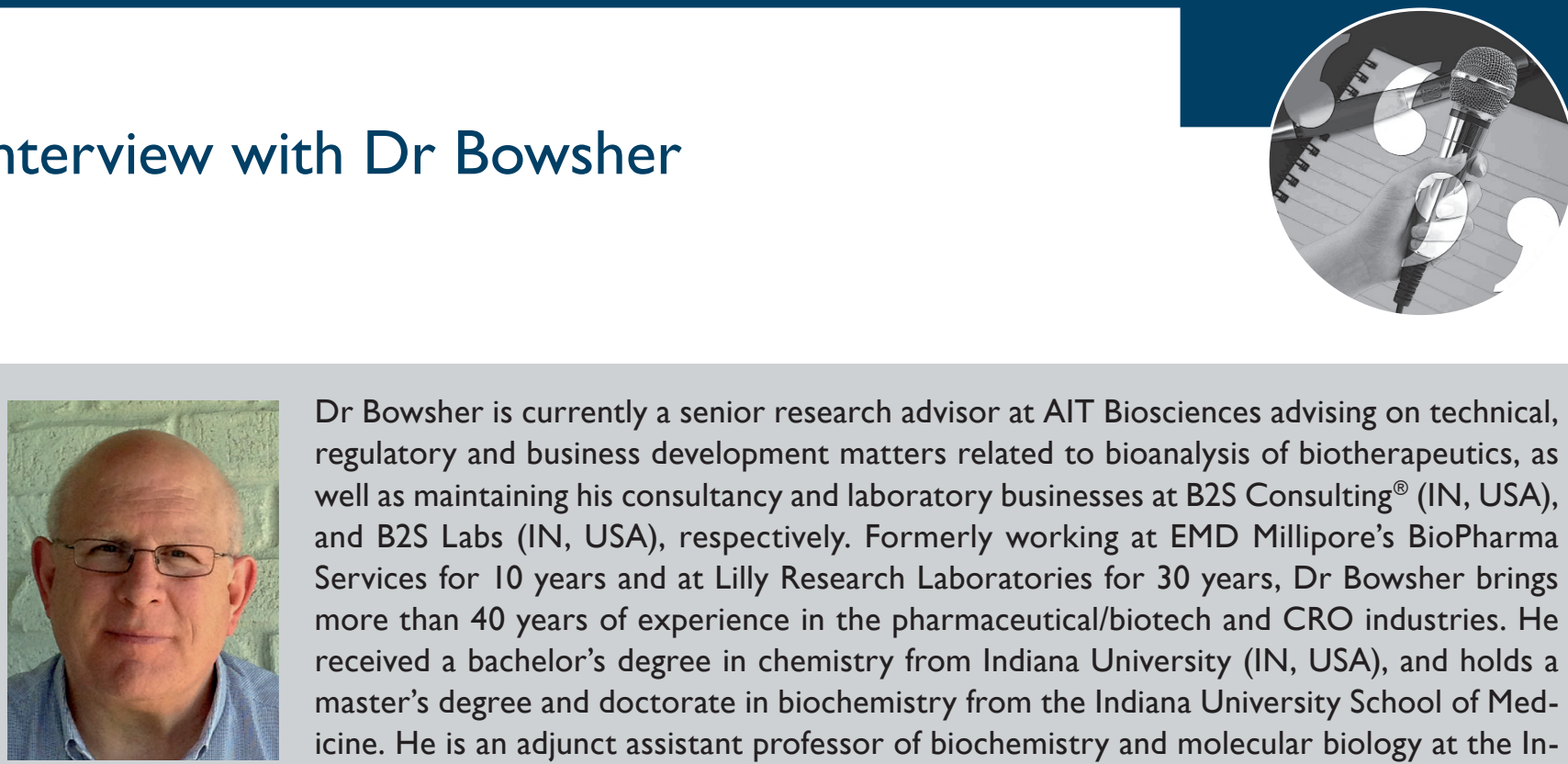

Dr Bowsher is currently a senior research advisor at AIT Biosciences advising on technical, regulatory and business development matters related to bioanalysis of biotherapeutics, as well as maintaining his consultancy and laboratory businesses at B2S Consulting ${ }^{\circledR}$ (IN, USA), and B2S Labs (IN, USA), respectively. Formerly working at EMD Millipore's BioPharma Services for 10 years and at Lilly Research Laboratories for 30 years, Dr Bowsher brings more than $\mathbf{4 0}$ years of experience in the pharmaceutical/biotech and CRO industries. He received a bachelor's degree in chemistry from Indiana University (IN, USA), and holds a master's degree and doctorate in biochemistry from the Indiana University School of Medicine. He is an adjunct assistant professor of biochemistry and molecular biology at the Indiana University School of Medicine. Since 1980, he has published more than 100 research papers and monographs, and 105 abstracts, as well as holding three patents. In 200I, Dr Bowsher founded and organized the Ligand Binding Assay Bioanalytical Focus Group (LBABFG) within the American Association of Pharmaceutical Scientists (AAPS) and in 2004 he was elected as an AAPS Fellow, as well as being awarded an AAPS Presidential Citation for his work in establishing an educational program, and has served as the co-organizer for the past 6 years of the highly successful AAPS Immunogenicity Training Course. In 20I2, Dr Bowsher received the Distinguished Alumnus Award, School of Science, Indiana University-Purdue University (IN, USA).

In March 2013, Dr Bowsher spoke to Alexandra Sklan at Bioanalysis regarding his bioanalytical career, involvement in the AAPS and the future outlook relating to macromolecule bioanalysis.

His primary research interests are the development and validation of ligand binding assays for the bioanalysis of macromolecules, endogenous compounds and conventional xenobiotic drugs to support regulatory-compliant assessments of PK/TK, PD and safety, and the ADME of biotherapeutics.

Could you tell Bioanalysis a little about your career to date \& how you ended up at AIT Biosciences?

The best metaphor for my career is a book that consists of several chapters. The first one would be represented by my 30 year career at the Lilly Research Laboratories, which began in 1973; while at Lilly I worked in clinical and preclinical drug metabolism, and diagnostic and experimental medicine. In the early 1980 s three different serendipitous factors led me into a career with a focus in bioanalysis and ligand binding assays (LBAs). First was my interest in analytical biochemistry, protein chemistry and in vivo metabolism. Second was the need to quantify drugs at low concentrations to support PK assessments in the early days before the availability of LC-MS as a routine bioanalytical tool. Interestingly, I spent the majority of my time in the early years developing radioimmunoassays to support the PK of conventional small-molecule drug candidates, not proteins. Third was Lilly's early entry into the field of biotechnology in the late 1970s, and which began with the manufacturing of the first rDNA derived biotherapeutic, human insulin, and was followed by a steady stream of other proteins and protein analogs. LBAs, primarily radioimmunoassay, were the critical technology for supporting bioanalysis of these early biotherapeutic drug candidates. It was at this time in the 1980s and 1990s that I first encountered many of the bioanalytical challenges that were inherent in the application of LBAs for regulatory-compliant bioanalysis. I will always be grateful for my Lilly experience and the opportunity to work with so many talented people, including several individuals who I consider great mentors and to whom I am indebted. My philosophy has been to try and serve as a good mentor to new young scientists as a way to pay back the individuals who invested in me. I decided to close my Lilly book chapter in 2003 and chose to make a career change into the CRO industry. This decision was made because I recognized that LC-MS was the focus at most

\section{Ronald R Bowsher}

B2S Consulting, 6656 Flowstone Way, Indianapolis, IN 46237, USA E-mail: ronb@b2s-stats.com 
CROs, and there was a paucity of laboratories that had expertise and capacity in conducting LBAs. Thus, I joined LINCO Diagnostic Services (MO, USA) early in 2003 with the idea of creating a CRO whose core technology was LBAs. This business grew commensurate with the emergence of biotechnology derived therapeutics as an importance source of new drugs. LINCO was acquired and today is now a part of the BioPharma Services division of EMD Millipore. Over the decade, this organization has evolved, grown in size and added new capabilities. While I still have many close friends at Millipore, I chose to close this chapter and my formal relationship ended in 2012. My plan was to focus on my consulting business (B2S Consulting $^{\circledR}$ ), which was formed in 2002 along with two former Lilly colleagues, Wendell Smith and Rocco Brunelle, and to begin a laboratory (B2S Labs) by partnering with Dan Wierda, a former Lilly colleague, whose mission is the generation and life-cycle management of custom LBA reagents and assays. Unexpectedly, it was at this point that an old friend, Mike Evans, approached me about the opportunity to help AIT Bioscience (IN, USA) expand their range of bioanalytical capabilities by growing its LBA services to complement their LC-MS business. I accepted his offer and started a new chapter, as I saw this as another opportunity to join a great group of people and mentor a small, but talented, group of scientists. I joined AIT Bioscience in the third quarter of 2012 and currently serve as a Senior Research Advisor for LBAs. Currently, I split my time between B2S Consulting, B2S Labs, AIT Bioscience, family and grandkids.

Having worked for both the pharmaceutical \& CRO industries, what would you say the main challenges are in maintaining effective communication between the two?

While I believe everyone desires effective communication and strives to achieve it, I am not sure it always occurs between these entities. Moreover, I believe the nature of communications between pharma/biotech and CROs is evolving and getting more complex. Today, drug development is more decentralized, involves greater numbers of representatives/participants, is a global endeavor, and probably less personal due to the dynamic and varied nature of personnel who are involved in the drug development process. Clearly, organizations who master good communication both verbal and written, will be better positioned long-term to be more competitive and successful. In addition, I believe there is an opportunity here for CROs to innovate with respect to electronic tools as a means for enhancing communications with customers and increasing operational efficiency.

In 2001, you founded \& organized the Ligand Binding Assay Bioanalytical Focus Group within the AAPS to create a forum to address issues, seek harmonization \& promote education for the development, validation \& application of analytical methods for the bioanalysis of small molecules, biotechnology products \& biomarkers by ligand binding assay methodologies. What was the main driving force behind this decision?

This is an easy question. I had the opportunity to attend both Crystal City BMV meetings (VA, USA) in early 2000. I was struck by just how different the nature of the interactions was at these two meetings. At the January smallmolecule BMV meeting, many people knew each other, harmonized consensus was readily achieved on many topics and there was lots of discussion around specific bioanalytical details. In contrast, at the inaugural macromolecule March meeting, fewer people knew each other, there was much diversity in BMV practices and the broad range of topics impeded discussions on specific bioanalytical details. Consequently, it was readily apparent to me and others that we needed more BMV discussions and interactions in order to help our colleagues in biotech 'catch up' with our smallmolecule bioanalytical colleagues. I give lots of credit to the US FDA and AAPS for bringing biotech folks together as a way to spur evolution and gain harmonization in LBA BMV practices. I simply harnessed the enthusiasm by reaching out to some new acquaintances, namely Jeff Sailstad, Steve Swanson and Marian Kelley, to create the Ligand Binding Assay Bioanalytical Focus Group (LBABFG). It seems funny now, but our biggest concern at the time was identifying the required minimum number of members (50) needed to create a new AAPS Focus Group. It has turned out that beginning this focus group has been one of the most gratifying aspects of my career. It brought together many people from diverse groups who share a common interest in bioanalysis of biotechnology drugs, fostered a number of close personnel friendships and through the efforts of many people has culminated in numerous White Papers on important bioanalytical topics related to biotherapeutics, including PK assays, 
immunogenicity and biomarker assays. I believe the LBABFG also served to energize the AAPS Biotec section. Lots of credit goes to many people, as the success of the LBABFG has far exceeded my early vision and expectations.

What are current hot topics under discussion?

With respect to LBA bioanalysis, I believe the hot topics would include global regulatory harmonization, evolution in immunoaffinity LC-MS of proteins, continued evolution in antidrug antibodies and neutralizing antibody testing, and progress in the technology and tools for quantitative determination of novel biomarkers.

How important do you think committees such as these are in harmonizing validation practices for macromolecule bioanalysis?

I believe these committees have been exceedingly valuable over the past decade for advancing LBA BMV practices. The LBABFG has sponsored numerous committees that have culminated in a number of key White Papers for BMV of LBAs to support PK/TK [1], ADA testing [2-4], detection of neutralizing antibodies [5,6] and biomarker testing [7]. Today, I am seeing more White Papers in other areas of drug development. I believe this is evidence of the perceived value of this activity.

With other members of the LBABFG you created two training courses, one for the application of ligand binding assays to support biotherapeutic PK \& one for immunogenicity testing of antidrug antibodies. For this work you were awarded a Presidential Citation. In your opinion, how should bioanalytical societies adopt more educational tools?

What I believe is educational programs, such as these, are valuable to the field of bioanalysis, particularly for supporting biotechnology-derived therapeutics. I believe AAPS and other societies are attempting to address this by making webinars, computer-based training, online training and e-Courses, a strategic priority. Furthermore, I believe the need for educational opportunities will continue to grow over the near term with the increased emphasis in globalization of drug development. I have also come to appreciate that the creation of educational programs requires a continued investment of time, effort and resources, as the course content needs to evolve to be timely and relevant. In this regard, Marie Rock and I have attempted to update the course content of the AAPS immunogenicity training course each year to keep it current with respect to science, methods and regulatory perspective.

In a recent Editorial in Bioanalysis, you suggest that the publication of White Papers will help govern regulatory issues in the bioanalytical standardization and development use of 'pharmaceutical-grade' kits as biomarker tools to support drug-development [8]. What role do you think guidance documents such as these play in reaching out to the wider bioanalytical community?

What I know is there is broad interest in biomarker quantification and the use of biomarker data to improve the success rate and cost-effectiveness of rational drug development. Without question, the use of research-use-only kits has grown steadily of the past couple of decades as pharma/biotech organizations have sought to use commercial kits as tools for performing quantitative determination of biomarkers. Despite their popularity and perceived convenience, there is not yet a broad consensus on what a priori constitutes acceptable kit design and performance. We believe White Papers offer a mechanism to share a collective opinion and perspective for communicating what we believe represents BMV best practices. This represents an important first step towards increasing harmonization in biomarker BMV practices, as exemplified by the broad initial impact of the publication by Lee et al. [7]. Furthermore, we hope to articulate what we believe are minimal expectations for acceptable kit design, method performance and documentation to aid kit manufacturers with creation of 'pharma-grade' kits. The availability of pharma-grade biomarker kits combined with harmonized BMV practices would result in increased productivity, decreased costs and provide greater consistency and reliability in biomarker data to support drug submissions.

What emerging areas will be seen in bioanalysis in years to come?

I believe we will continue to witness a merging of LBA technology and LC-MS for quantitative determination of biotherapeutics. At the same time, we are seeing a steady stream of innovation in the design and delivery of biotech drugs. This will help ensure we have new and evolving bioanalytical challenges in the near term. 
In addition, I believe we are seeing evidence of more novel biological modalities, such as cellular therapies, enter drug testing. These innovative therapies will create new opportunities for BMV innovation. A common requirement for all these LBA-based technologies is the need for high quality biological reagents, such as antibodies. Broad availability of technology for systematic generation and optimization of custom antibodies would be a 'game-changer' for biotech bioanalysis, because of the universal requirement for these unique reagents.

Do you have any advice for those looking to start a career in the bioanalytical field?

This is probably the hardest question you have asked. Because of the rapidly evolving nature for changes in how pharma/biotech $R \& D$ is being conducted, it's difficult for me to reliably project out much beyond approximately 5 years. For this reason, I would advise a young person with an interest in bioanalysis to gain experience in both biological reagent-based methods (i.e., LBAs) and LC-MS/MS technology. It also seems that there is now an opportunity for individuals who have expertise that allows them to work at the interface between these different bioanalytical disciplines. Lastly, I would strongly encourage someone who is interested in bioanalysis to develop good communications skills, both verbal and written, because success in this global business demands clear, concise and effective communication.

\section{Disclaimer}

The opinions expressed in this interview are those of the interviewee and do not necessarily reflect the views of Future Science Ltd.

Financial \& competing interests disclosure The interviewee has no relevant affiliations or financial involvement with any organization or entity with a financial interest in or financial conflict with the subject matter or materials discussed in the manuscript. This includes employment, consultancies, honoraria, stock ownership or options, expert testimony, grants or patents received or pending, or royalties.

No writing assistance was utilized in the production of this manuscript.

\section{References}

1 DeSilva B, Smith W, Weiner R et al. Recommendations for the bioanalytical method validation of ligand-binding assays to support pharmacokinetic assessments of macromolecules. Pharm. Res. 20(11), 1885-1900 (2003).

2 Mire-Sluis AR, Barrett YC, Devanarayan V et al. Recommendations for the design and optimization of immunoassays used in the detection of host antibodies against biotechnology products. J. Immunol. Methods 289(1-2), 1-16 (2004).

3 Shankar G, Devanarayan V, Amaravadi L et al. Recommendations for the validation of immunoassays used for detection of host antibodies against biotechnology products. J. Pharm. Biomed. Anal. 48(5), 1267-1281 (2008).

4 Ponce R, Abad L, Amaravadi L et al. Immunogenicity of biologically-derived therapeutics: assessment and interpretation of nonclinical safety studies. Regul. Toxicol. Pharmacol. 54(2), 164-182 (2009).

5 Gupta S, Indelicato SR, Jethwa V et al. Recommendations for the design, optimization, and qualification of cell-based assays used for the detection of neutralizing antibody responses elicited to biological therapeutics. J. Immunol. Methods 321(1-2), 1-18 (2007).

6 Gupta S, Devanarayan V, Finco D et al. Recommendations for the validation of cell-based assays used for the detection of neutralizing antibody immune responses elicited against biological therapeutics. J. Pharm. Biomed. Anal. 55(5), 878-888 (2011).

7 Lee JW, Devanarayan V, Barrett YC et al. Fit-forpurpose method development and validation for successful biomarker measurement. Pharm. Res. 23(2), 312-328 (2006).

8 Bowsher RR, Nowatzke W, Sailstad JM, Khan MU. Application of commercial research-grade biomarker assays in drug development: is it time to create 'pharmaceutical-grade' kits? Bioanalysis 4(20), 2427-2430 (2012). 